\title{
WIND FEATURES AND WIND VELOCITIES
}

\author{
Michael Grewing \\ Astronomisches Institut der Universität \\ Waldhäuserstrasse 64, D-7400 Tübingen, Germany, Fed.Rep.
}

\begin{abstract}
.
Fast winds have been detected in the spectra of many nuclei of planetary nebulae (PNNi). The wind velocities range from about $600 \mathrm{~km} / \mathrm{s}$ to roughly $4000 \mathrm{~km} / \mathrm{s}$. While these winds add little to the mass of the nebular shells they may significantly effect their internal kinematics.

By studying the emission from the faint outer envelopes of $\mathrm{PNe}$ one can infer also the wind properties of the progenitors of the current nuclei. This will in the end allow to test quantitatively current models of the origin of PNe.
\end{abstract}

\section{INTRODUCTION}

The idea that stellar winds could play a significant role in planetary nebulae (PNe) arose apparently first from theoretical considerations in order to explain the shell-type structure of some of the objects. Mathews (1966) calculated the first models assuming that such winds would have velocities of several $10^{2} \mathrm{~km} / \mathrm{s}$, and that the mass loss rates would be in in the order of $\mathrm{dM} / \mathrm{dt} \sim 10^{-5} \mathrm{M}_{\mathrm{o}} / \mathrm{yr}$. It was later shown that the morphology of PNe can also be explained by an initial pressure gradient (Sofia and Hunter 1968), and radiation pressure acting on dust particles (Ferch and Salpeter 1975). Still, the idea that stellar winds play a decisive role in the evolution of $\mathrm{PNe}$ has gained new momentum since such winds have actually been detected.

\section{WINDS FROM PLANETARY NUCLEI}

Among the first measurements carried out with the International Ultraviolet Explorer (IUE) were observations of planetary nebulae and their nuclei. Immediately, evidence was found for the presence of stellar winds : the UV lines of $\mathrm{N} \mathrm{V,O} \mathrm{V,} \mathrm{C} \mathrm{IV,} \mathrm{Si} \mathrm{IV}$ and others were seen to show the characteristic P Cygni profiles (Heap et al. 1978. A large number of such spectra has been compiled by Heap (1983). In Fig. 1 we show a few examples. 

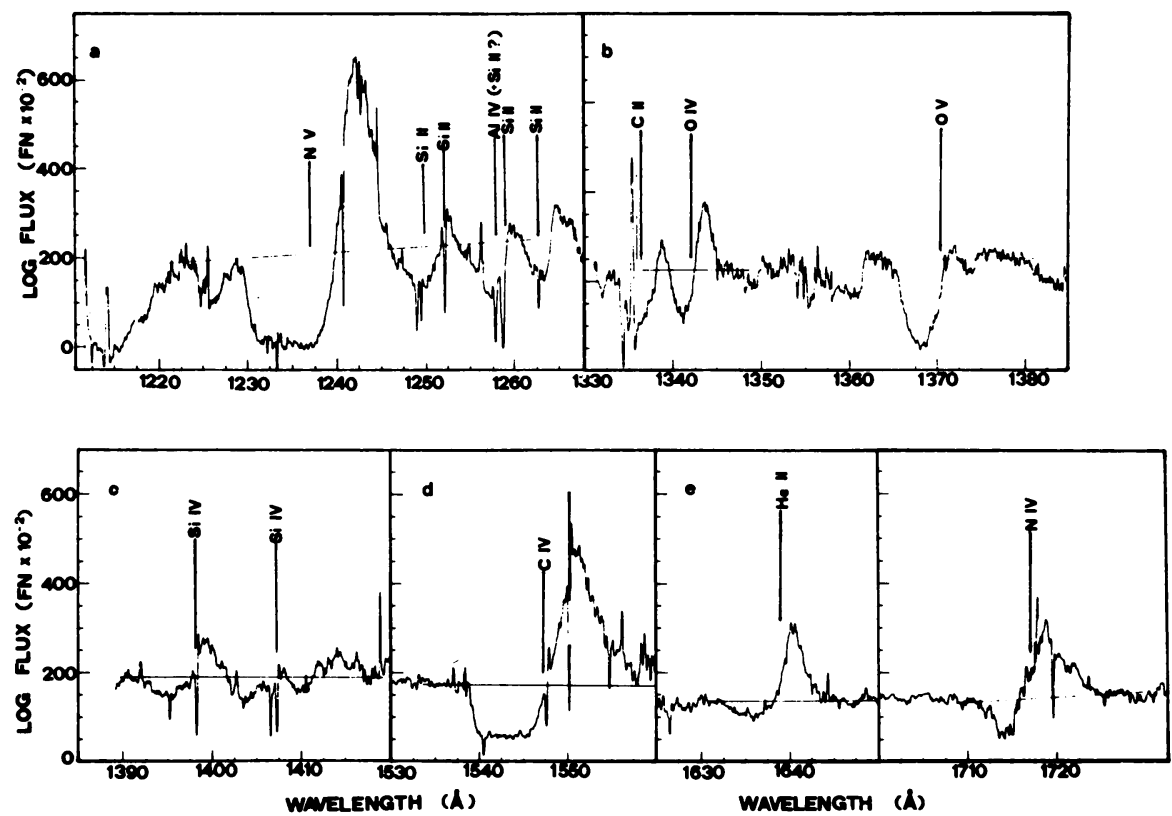

Fig. 1 : Evidence for a fast stellar wind in the high-resolution IUE spectrum of NGC 6543 (from Bianchi, Cerrato, and Grewing 1986).

While Fig.1 taken from Bianchi et al.(1986) is based on high-resolution (0.2 $\AA$ )spectra obtained with the IUE, most of the initial studies of winds in PNe relied on the much easier to obtain low-resolution spectra (6 $\AA)$. Perinotto (1983) and Cerruti-Sola and Perinotto (1985) collected such spectra for 60 PN nuclei (PNNi), 42 of which showed a measurable continuum. In 22 out of these cases $P$ Cygni lines appear, giving clear evidence for the presence of a wind. Based on the location of these objects in the Hertzsprung-Russell diagram, Cerruti-Sola and Perinotto suggested that the occurence of a wind may actually be correlated with the temperature and radius of the stars. Indeed, by studying separately nuclei with WR, Of-Ofp, WR-Of, C-N, O-sdO, and continuumtype spectra, evidence for a wind was found in $100 \%, 90 \%, 100 \%, 70 \%, 40 \%$, and $30 \%$ of the cases, respectively, where one must note, however, that the numbers quoted are highly uncertain due to the small numbers of objects available in each subclass.

In many cases, the IUE low-resolution spectra have been used to determine wind velocities. A list is given in the paper by Cerruti-Sola and Perinotto (loc.cit.). Already from these studies it is clear that the original conjecture of wind velocities of only a few $10^{2}$ $\mathrm{km} / \mathrm{s}$ was too pessimistic by almost an order of magnitude. This is substantiated by the much more precise measurements of wind terminal velocities from high-resolution spectra as performed by Heap (1986). She has analysed such 30 times better resolved spectra from 22 objects, and we have studied some additional PNNi at high resolution at Tübingen. Based on these results, we have computed the histogram of the frequency of 
occurence of certain wind velocities as shown in Fig. 2. The fact that this analysis is based on only 20 objects (because the spectra of some PNNi show no evidence for P Cygni lines) limits, of course, the statistical significance of the result. Still, I think, the important message is that the observed wind velocities range from about $600 \mathrm{~km} / \mathrm{s}$ up to about $4000 \mathrm{~km} / \mathrm{s}$, i.e. they extend over a very large range.

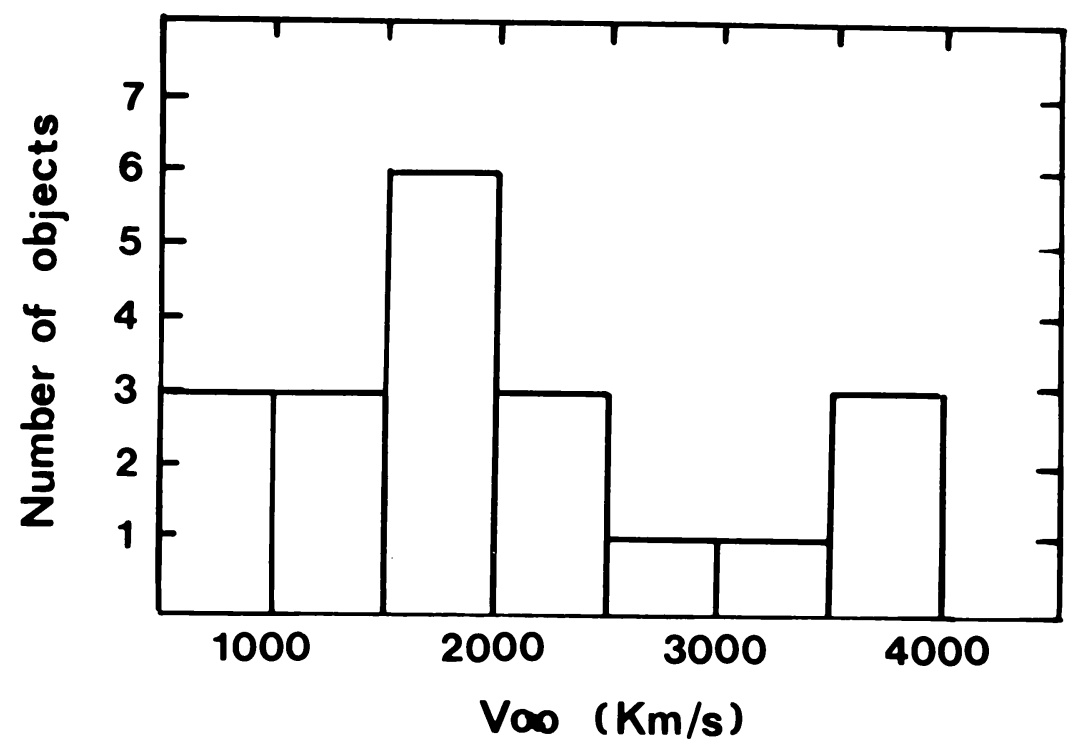

Fig. 2 : Histogram of the observed terminal wind velocities based on data from a total of 20 objects.

This result immediately leads to the question : what is driving the winds ? If we assume as a hypothesis that it is the radiation pressure from the central star, then we can use the relation (see Abbott 1982)

$$
\mathrm{v}_{\infty}=(\alpha / 1-\alpha)^{1 / 2} \mathrm{v}_{\mathrm{esc}}=774\left[(\mathrm{M} / \mathrm{R})\left(1-\mathrm{L} / \mathrm{L}_{\mathrm{Edd}}\right)\right]^{1 / 2}[\mathrm{~km} / \mathrm{s}] \text {. }
$$

Using $L=4 \pi R^{2} \& T_{\text {eff }}{ }^{4}$ and $L=6 \cdot 10^{4}(M-0.50)$, with $M, L$ and $R$ in solar units, we obtain

$$
\mathrm{V}_{\infty}=6.85 \cdot 10^{-3} \mathrm{~T}_{\text {eff }}\left[\mathrm{M}\left(1-\mathrm{L} / \mathrm{L}_{\text {Edd. }}\right)\right]^{1 / 2}[\mathrm{M}-0.50]^{-1 / 4}[\mathrm{~km} / \mathrm{s}] \text {. }
$$

Such a relation can be tested observationally as shown e.g. by Heap (1986). In Fig. 3a,b we have taken a similar approach by plotting the observed terminal velocities as a function of the PNNi's temperature and as a function of the excitation class of the corresponding $\mathrm{PNe}$, respectively. In producing Fig. 3a we have made a special attempt to use the most accurate temperature determinations available rather than the Zanstra temperatures used in the original study by Heap (loc.cit.). However, irrespective of such detail, all these diagrams show a correlation as expected from the theory of radiation pressure driven winds. Furthermore, as pointed out by Heap, they show that the range over which the masses of the nuclei scatter seems to be very limited. The fit through the 
data points as given in Heap (1986) suggests a mean value of $0.60 \mathrm{M}_{\mathrm{o}}$ with very little scatter indeed.
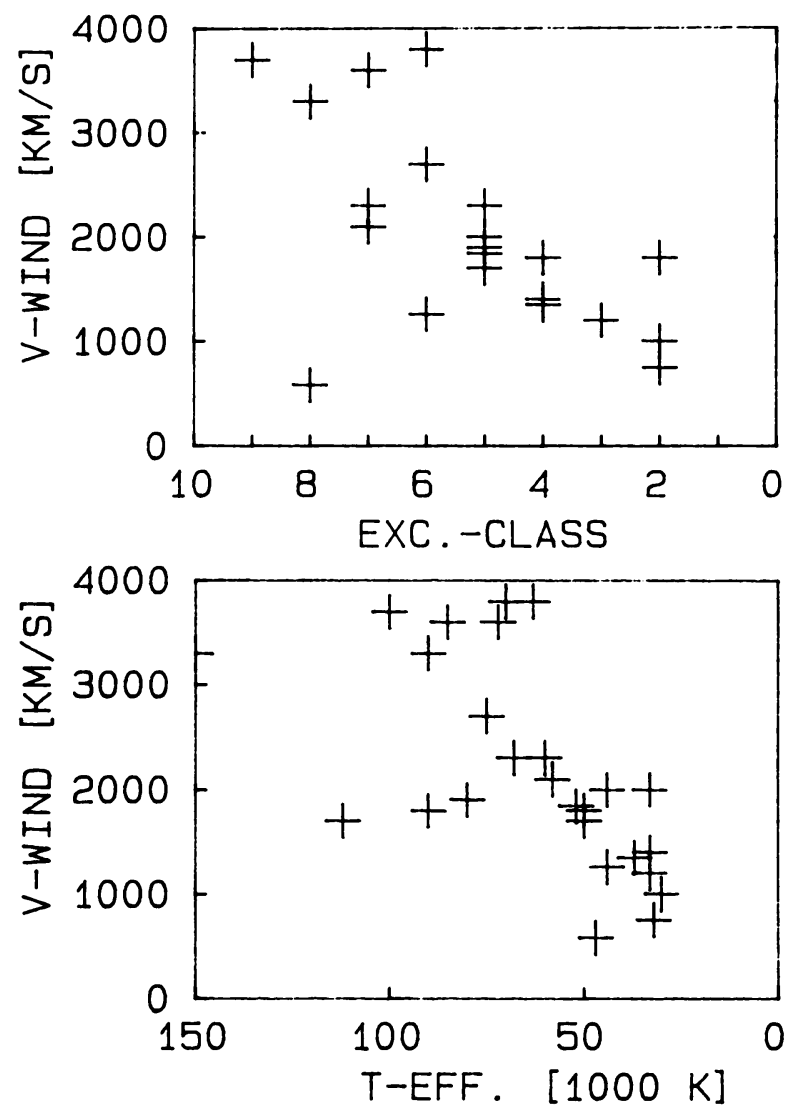

Fig. 3 : Observed terminal wind velocities plotted as a function of the temperature of the central stars (upper plot), and the excitation class of the surrounding nebulae (lower plot).

I want to point out in passing that the greatly improved atmosphere calculations carried out by Mendez et al. (1987) will probably give even better agreement between observations and theoretical predictions for radiatively driven winds.

Turning now to the physical state of these fast winds, i.e. their density, temperature, and ionization, it is somewhat surprising to see how little is known about these parameters. Obviously, such studies would need IUE high-resolution spectra, and those have until now been analysed in detail only for a very small number of objects. I would like to draw attention in particular to the work of Bombeck et al.(1986) in which the authors analyse the column densities of various wind ions (normalized with respect to $N(O V)$ ) and seem to find some weak correlation with the excitation class of the nebulae. This 
parameter is taken as a measure of the temperature of the central stars. Bombeck et al. find the winds to be more highly ionized for the high excitation class objects, suggesting photoionization as the main ionization mechanism in the winds. Instead of using column densities, we have collected from the literature total optical depth measurements from the P Cygni lines of several wind ions. In Fig. 4 these are plotted as a function of the excitation class of the surrounding nebulae, and we find the same kind of trend as suggested in the paper quoted, i.e. the degree of ionization seems to increase with increasing excitation class but we have far too few data to arrive at any final conclusion. Obviously, it would be extremely worthwhile to extend such studies to many more objects by analysing in a systematic way the high-resolution spectra available from the IUE archive.

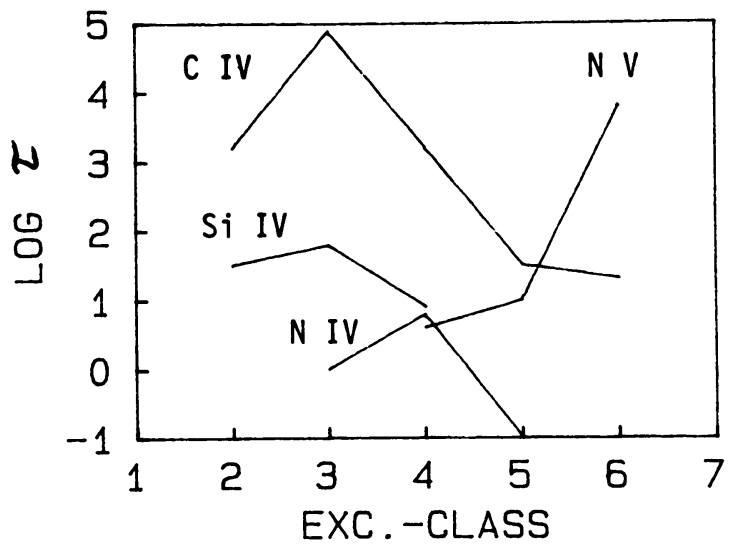

Fig. 4 : Measured total optical depths for several wind ions plotted as a function of the excitation class of the surrounding nebulae. The data pertain to NGC 6572, IC 418, IC 2149, and IC 3568 .

Let me now briefly turn to the issue of how the presence of a fast wind from the central star may affect the surrounding nebular shells. I would first like to refer to the work of Adam and Köppen (1985) who computed models for the two objects NGC 4361 and NGC 1535. The interesting point of this comparative study is that the IUE spectra show clear evidence for a fast wind in the case of NGC 1535 but no such evidence for NGC 4361. The authors had chosen these particular objects because Mendez et al. (1981) had analysed the spectra of the central stars, thus providing a detailed model for the photon flux from the central objects. Using this as input in the calculations of the nebular ionisation, Adam and Köppen found consistent results for NGC 4361 but no consistency for NGC 1535. In the latter case there was a shortage of $\mathrm{He}^{+}$ionizing photons which they were able to overcome, however, by taking into account photons emitted from the fast wind which they assumed to be hot $\left(\log \mathrm{T}_{\mathrm{e}}=5.5\right)$.

Given the rapid improvement of self-consistent model atmophere calculations that will in the future also include the extended wind spheres, we will probably soon know if the fast wind plasma is as hot as assumed by Adam and Köppen or if it is considerably cooler. The few observations at $x$-ray wavelengths that have been published so far seem 
to suggest that the winds may indeed not be very hot. While the ionizing effect of the fast winds remains an open issue for the the time being, there is accumulating evidence that the winds cause dramatic effects by dynamically interacting with the material that exists around the central stars from previous wind episodes. There is an increasing number of papers in the literature and also a large number of poster contributions at this conference which deal with peculiar outflow phenomena in PNe which occur at velocities of several hundred $\mathrm{km} / \mathrm{s}$, i.e. velocities which are an order of magnitude larger than typical nebular expansion velocities.

The Eskimo nebula NGC 2392 is one of the objects studied in this context. O'Dell and Ball (1985) were the first to report very high outflow velocities which they found after carefully subtracting the spectrum of the central star. Gieseking et al. (1985) found emission from material moving at about $200 \mathrm{~km} / \mathrm{s}$ at a position angle of roughly $70^{\circ}$. In the poster contribution by Phillips et al.(1987), this result is basically confirmed but their interpretation differs somewhat from that of Gieseking et al..

Since these early findings, Gieseking and Solf (1986) reported similar results for NGC 6751. In Table $1 \mathrm{I}$ have tried to summarize the current situation, making heavy use of a recent compilation of kinematical data by Weinberger (1987).

Whereas the fast flow phenomena seem to occur in places where the fast inner wind is breaking through the nebular shells and carrying away individual pockets of gas, recently some very interesting results have been reported which may relate to cases where the fast winds get stopped and conpacted at the inner edge of the nebular shells. In analysing the IUE high-resolution spectrum of NGC 40, Bianchi and Grewing (1987) saw narrow C II absorption which was clearly not of interstellar origin and which they suggested could arise at the interface between the fast wind coming from the central star and the nebula proper. This argument was based both on the velocity and of the column density of the absorbing material. Still much more intriguing are the findings reported recently by Kaler et al. (1987) for Abell 78. These authors see a number of narrow absorption lines superimposed on the broad $P$ Cygni profiles and the continuum. They can clearly be distinguished from the interstellar lines which are also present, and they may -as the authors suggest- originate at the interface of the fast wind with the nebula.

While I have so far addressed winds ejected by the current nuclei, I would now like to briefly turn to winds from their progenitors. I think that there is a general consensus that the extended halos found in about $2 / 3$ of all PNe searched for faint outer shells (see e.g. Chu et al. 1987) must have originated from the AGB winds of the progenitors.

As the halo gas has been decelerated to velocities of less than $10 \mathrm{~km} / \mathrm{s}$, high spectral resolution is required to study the kinematics. In view of the low surface brightness this is a very demanding requirement that has so far limited the studies to only a few objects. Fig. 5 shows the results of Hippelein et al. (1985) who have studied the three objects NGC 6543, NGC 6826, and NGC 7662, finding velocities which are indeed consistent with the AGB wind origin of the material. 
Table 1

Planetary Nebulae with Large Internal Motions

\begin{tabular}{ccl}
\hline \multicolumn{1}{c}{ PK } & Name & References \\
\hline $10+18.2$ & M 2-9 & AS72, Wa81, CS83, Ca83,PMR87 \\
$10+0.1$ & NGC 6537 & BeS83, PMR87 \\
$36-1.1$ & Sh $2-71$ & PMR87 \\
$36-57.1$ & NGC 7293 & MeW80, WM87 \\
$81-14.1$ & A 78 & MPM87, KFSH87 \\
$83+12.1$ & NGC 6826 & BIP87 \\
$86-8.1$ & Hu $1-2$ & PMR87 \\
$89+0.1$ & NGC 7026 & PMR87 \\
$96+29.1$ & NGC 6543 & Mu68, BIP87 \\
$106-17.1$ & NGC 7662 & BIP87 \\
$107+2.1$ & NGC 7354 & BIP87 \\
$130-11.1$ & M $1-1$ & ST87 \\
$197+17.1$ & NGC 2392 & RAT83, ODB85, GBS85,BIP87,PMR87 \\
$208+33.1$ & A 30 & JCh87 \\
$234+2.1$ & NGC 2440 & KA74, WH87 \\
$261+32.1$ & NGC 3242 & BIP87 \\
$277-3.1$ & NGC 2899 & LFRR87 \\
$315-0.1$ & He 2-111 & We78 \\
$331-1.1$ & Mz 3 & LM83, WH87 \\
$349+1.1$ & NGC 6302 & MJ67, MW80, DBABT83, WH87 \\
$359-0.1$ & Hb 5 & PMR87
\end{tabular}

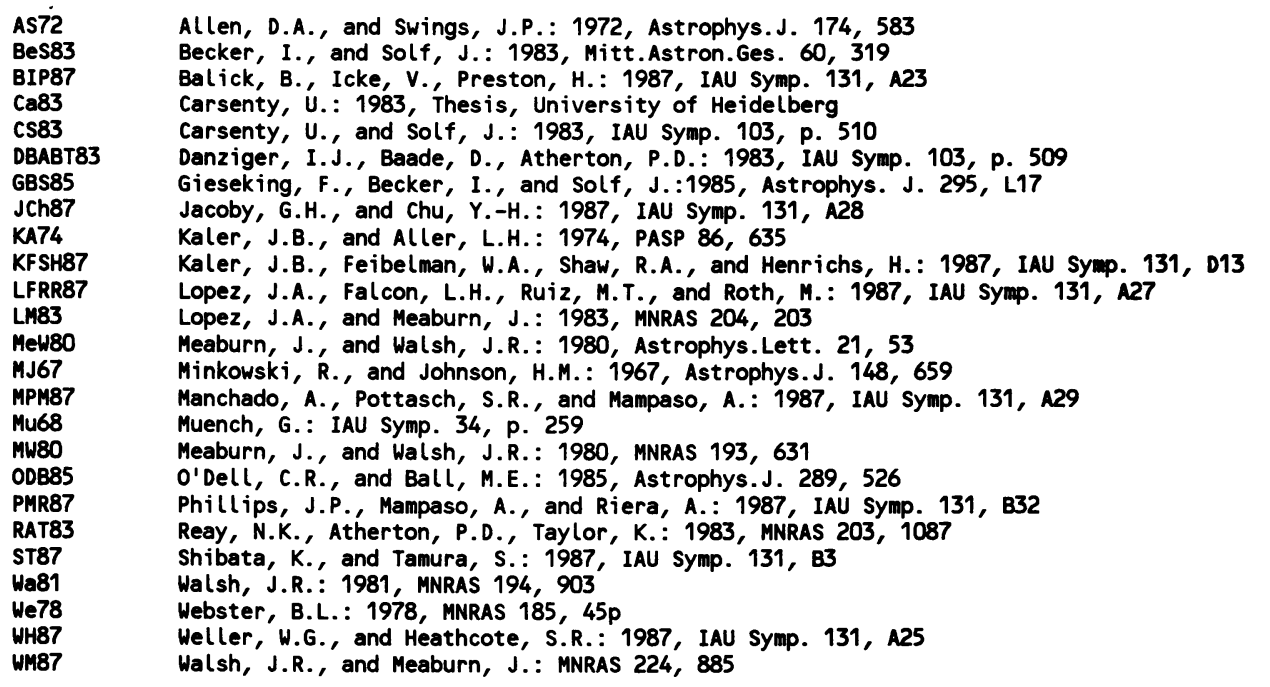




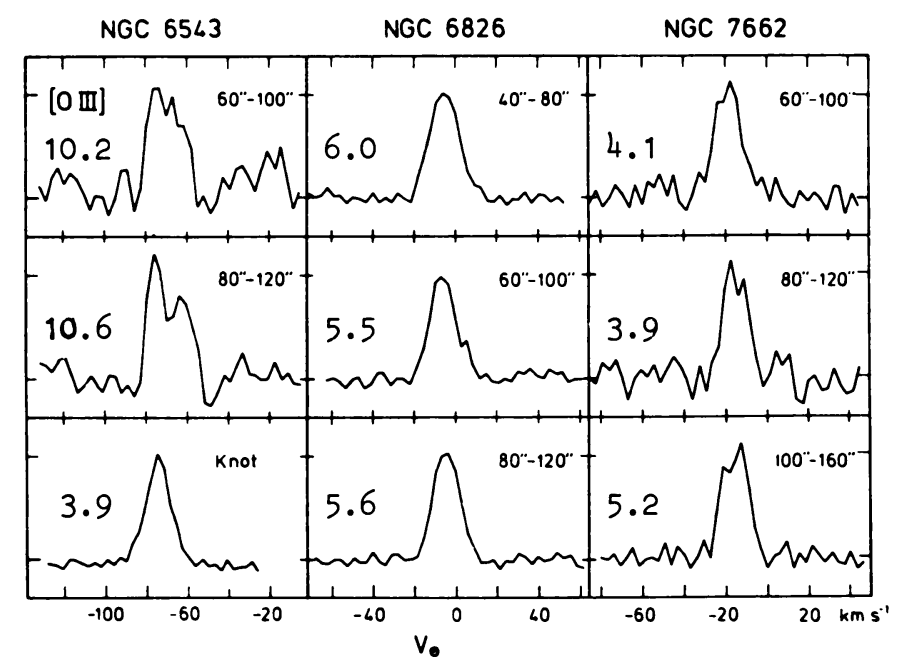

Fig. 5 : [O III] emission line profiles from the halo of NGC 6543 , NGC 6826 , and NGC 7662, respectively. The numbers in each plot give the size of the annular diaphragms used to observe the halo emission. Still, a correction had to be made for straylight from the bright nebular shells (see Hippelein, Baessgen and Grewing 1985 for details). Also, in each plot the expansion velocity of the halo gas is given in $\mathrm{km} / \mathrm{s}$.

Since then we have obtained a number of absolutely calibrated optical spectra which reveal the ionization structure of the nebulae, and, more importantly in the present context, yield densities in the outer wind spheres as a function of radius. This allows several tests of the AGB wind model. Firstly, we can check the $r^{-2}$ dependence which is expected for a wind expanding at constant velocity $v$. Secondly, we can extrapolate the halo densities inward to those radii where the bright nebular shells are located and determine the ratio of the current nebular density to the extrapolated halo density. This should be a measure of the compression factor if we adopt e.g. the two-wind model of $\mathrm{K}$ wok et al. (1978). It is interesting to note that except for two objects, the density ratio significantly exceeds the adiabatic ratio, suggesting instead isothermal shocks.

Finally, we can derive the ratio $(\mathrm{dM} / \mathrm{dt}) / \mathrm{v}$. Results have been given by Bässgen et al.(1987) and are repeated in Table 2.

To derive $d M / d t$, we would need to know the expansion velocity $\mathbf{v}$ but the resolution of our spectra is not sufficient to actually measure v. Still, a reasonabe estimate of the mass loss rate of the progenitors can be obtained by assuming $\mathrm{v}<10 \mathrm{~km} / \mathrm{s}$. Typical values thus derived are in the range $210^{-6}$ to $210^{-5} \mathrm{M}_{\mathrm{o}} / \mathrm{yr}$, values that are not unreasonable for AGB stars. Still, they seem to be lower than the values found recently by Taylor et al. (1987) who analysed the radio continuum spectra of compact PNe. By fitting the observations with theoretical spectra derived from free-free emission models for photonlimited, ionized shells corresponding to stellar-wind type envelopes, Taylor et al. come to the conclusion that for the objects of their sample $(\mathrm{dM} / \mathrm{dt}) \geqslant 10^{-5} \mathrm{M}_{\mathrm{o}} / \mathrm{yr}$. 
Table 2

Halo density as a function of angular radius

\begin{tabular}{|c|c|c|c|c|}
\hline Object & adopted distance & Pos. & density & $\begin{array}{l}\text { corresp.M/V } \\
\mathrm{cm}^{-3}(\mathrm{Mo} / \mathrm{y})(\mathrm{km} / \mathrm{s}\end{array}$ \\
\hline NGC 3132 & $670 \mathrm{pc}$ & $\begin{array}{l}30 " \\
54 " \\
\end{array}$ & $\begin{array}{l}190 \\
40 \\
\end{array}$ & $\begin{array}{l}7.0 \\
4.5 \\
\end{array}$ \\
\hline NGC 1535 & $1500 \mathrm{pc}$ & $\begin{array}{l}31 " \\
40^{\prime \prime}\end{array}$ & $\begin{array}{l}16 \\
11\end{array}$ & $\begin{array}{l}3.0 \\
3.5\end{array}$ \\
\hline IC 418 & $1400 \mathrm{pc}$ & $\begin{array}{l}15^{\prime \prime} \\
29 "\end{array}$ & $\begin{array}{l}200 \\
40\end{array}$ & $\begin{array}{l}8.0 \\
6.0 \\
\end{array}$ \\
\hline NGC 2452 & $4000 \mathrm{pc}$ & $\begin{array}{l}11^{\prime \prime} \\
17^{\prime \prime}\end{array}$ & $\begin{array}{l}100 \\
30\end{array}$ & $\begin{array}{l}17.0 \\
11.0\end{array}$ \\
\hline IC 2448 & $2900 \mathrm{pc}$ & $\begin{array}{l}17 " \\
29 " \\
\end{array}$ & $\begin{array}{l}11 \\
3:\end{array}$ & $\begin{array}{l}2.5 \\
2.0 ; \\
\end{array}$ \\
\hline NGC 2867 & $1600 \mathrm{pc}$ & $\begin{array}{l}17 " \\
34^{\prime \prime}\end{array}$ & $\begin{array}{l}25 \\
6 \\
\end{array}$ & $\begin{array}{l}1.8 \\
1.8 \\
\end{array}$ \\
\hline IC 2165 & $2400 \mathrm{pc}$ & $\begin{array}{l}10^{\prime \prime} \\
17^{\prime \prime} \\
27^{\prime \prime}\end{array}$ & $\begin{array}{l}66 \\
23 \\
9 \\
\end{array}$ & $\begin{array}{l}3.5 \\
3.3 \\
4.0 \\
\end{array}$ \\
\hline
\end{tabular}

\section{CONCLUSIONS}

Stellar winds from the nuclei of planetary nebulae are now a well established phenomenon that occurs in a large fraction of the objects. The diagnostically most valuable information about such winds comes from high-resolution UV spectra obtained with the IUE satellite. The measured wind velocities range from about $600 \mathrm{~km} / \mathrm{s}$ to more than $4000 \mathrm{~km} / \mathrm{s}$. The can be accounted for by models of radiation pressure driven winds.

The mass carried away by these winds is far too little to significantly add to the mass of the nebular shells. Still, the fast winds could have an ionizing effect, and they almost certainly cause dramatic kinematical effects as they interact with the ambient medium. They may indeed be responsible for the peculiar motions at several $10^{2} \mathrm{~km} / \mathrm{s}$ observed now in a number of objects, often in the form of bipolar flows.

From detailed studies of the gas in the faint outer shells of $\mathrm{PNe}$, a very common phenomenon as recent studies with modern electronic detectors have shown, it seems now also possible to determine the properties of the AGB winds from the progenitors of the current nuclei thereby allowing to test models for the origin of planetary nebulae. While still at an early stage, these studies may in the end enable us to determine quantitatively the role that winds play during the various phases in the late stages of stellar evolution to accumulate the amount of material that we now find present in the different nebular shells.

Acknowledgement: I would like to thank M.Bässgen and S.Cerrato for their help in preparing the final version of this manuscript. 


\section{REFERENCES}

Adam, J., and Köppen, J.: 1985, Astron.Astrophys. 142, 461

Abbott, D.C.: 1982, Astrophys.J. 259, 282

Bässgen, M., Bässgen, G., Barnstedt, J., Grewing, M., Bianchi,L.

Mitt. Astron.Ges. 67,342

Bianchi, L., Cerrato, S., and Grewing, M.: 1986, Astron. Astrophys. 169, 227

Bianchi, L., and Grewing, M.: 1987, Astron.Astrophys. 181,85

Bombeck, G., Köppen, J., and Bastian, U.: 1986, in 'New Insights in Astrophysics', ESA SP - 263, p.287

Cerruti-Sola, M., and Perinotto, M.: 1985, Astrophys.J. 291, 237

Chu, Y.-H., Jacoby, G.H., and Arendt, R.: 1987, Astrophys.J.Suppl.Ser. 64, 529

Ferch, R.L., and Salpeter, E.E.: 1975, Astrophys.J. 202, 195

Gieseking, F., Becker,I., and Solf, J.: 1986, Astrophys.J. 295, L17

Gieseking, F., and Solf, J.: 1986, Astron.Astrophys. 163, 174

Heap, S.R., Bogess, A., Holm, A., Klinglesmith, D.A., Sparks, W., West, D., Wu, C.C.,

Boksenberg, A., Willis, A., Wilson, R., Macchetto, F., Selvelli, P.L., Stickland, D.,

Greenstein, J.L., Hutchings, J.B., Underhill, A.B., Viotti, R., and Whelan, J.A.J.: 1978, Nature 275, 385

Heap, S.: 1983, IAU Symp. 103, p. 375

Heap, S.: 1986, in 'New Insights in Astrophysics', ESA SP-263, p. 291

Hippelein, H.H., Bässgen, M., and Grewing, M.: 1985, Astron.Astrophys. 152, 213

Kaler, J.B., Feibelman, W.A., and Henrichs, H.F.: 1987, IAP 87-15 (Preprint)

Kwok, S., Fitzgerald, P.M., and Purton, C.R.: 1978, Astrophys.J. 219, L125

Mathews, W.G.: 1966, Astrophys.J. 143, 173

Mendez , R.H. , Kudritzki, R.P., Gruschinkske, J., Simon, K.P.: 1981,

Astron. Astrophys. 101, 323

Mendez , R.H., Kudritzki, R.P., Herrero, A., Husfeld, D., Groth, H.G.: 1987,

Astron. Astrophys. (in press)

O'Dell, C.R., and Ball, M.E.: 1985, Astrophys.J. 289, 526

Perinotto, M.:1983, IAU Symp. 103, 343

Phillips, J.P., Mampaso, A., Riera, A.: 1987,IAU Symp. 131, B32

Sofia, S., and Hunter, J.H.: 1968, Astrophys.J. 152, 405

Taylor, A.R., Pottasch, S.R., and Zhang, C.Y.: 1987, Astron.Astrophys. 171,178

Weinberger, R.: 1987, Preprint 